\title{
Power Law Dependencies to Detect Regions of Interest
}

\author{
Yves Caron, Harold Charpentier, Pascal Makris, and Nicole Vincent \\ Laboratoire d'Informatique, Université François Rabelais, \\ 64 avenue Jean Portalis, 37200 Tours, France \\ yves.caron@etu.univ-tours.fr, \{vincent,makris\}@univ-tours.fr
}

\begin{abstract}
This paper presents a novel approach to detect regions of interest in digital photographic grayscale images using power laws. The method is intended to find regions of interest in various types of unknown images. Either Zipf law or inverse Zipf law are used to achieve this detection. The detection method consists in dividing the image in several sub-images, computing the frequency of occurence of each different image pattern, representing this distribution by a power law model and classifying the sub-frames according to the power law characteristics. Both power laws models allow region of interest detection, however inverse Zipf law has better performances than Zipf law. The detection results are generally consistent with the human perception of regions of interest.
\end{abstract}

Keywords: Segmentation, region detection, region of interest, compression, coding.

\section{Introduction}

The detection of regions of interest in images is a difficult problem, which has many applications, such as object detection and recognition, image indexation and compression optimization. Our aim is to design a method which is generic enough to be able to detect regions of interest in various types of photographic static images without requiring previous knowledge of the scene or the objects to be detected. A possible application is to implement this function for automatic region of interest detection in a JPEG 2000 encoder. Unsupervised region of interest detection in unknown images is a difficult task since we cannot use color or shape information to find the objects of interest in the image. A possible approach is to use semi-local texture characteristics to detect parts of the image which have distinctive features from the surrounding background. Some existing works in this domain include Beaver et al. [1] who use a measure of fractal dimension to detect man-made objects in aerial images, Kadir and Brady [2] who use an entropy measure to detect salient regions in images, and Wang et al. [3] who use the statistical repartition of wavelet coefficient to extract foreground objects in photographic images. This paper presents a novel approach to detect region of interest by analyzing statistical distribution of image patterns using power laws. Power laws models such as Zipf law have been used in many domains, such as linguistics by Zipf [4], Miller and Newman [5] and Cohen et al.[6] by Hill [7] and Makse and al.[8] in urban population studies, by Mantegna and al. [9] in the sequencing of the human genome, or by Breslau et al. [10] and Huberman [11] in Internet 
traffic analysis. It has also been used in the domain of image analysis by Vincent et al. [12] for compression quality evaluation and by Caron et al. [13] for detecting artificial objects in natural environments. The problem of region of interest detection can be considered as a generalization of the object detection problem. In this paper we will first present the power laws models used, namely Zipf law and inverse Zipf law, then we will present how they can be used for image analysis. The detection method will be detailed and experimental results will be presented.

\section{Power Law Models}

\subsection{Zipf Law}

This law was determined empirically in 1949 by G.K Zipf [4]. It states that in a topologically organized set of symbols, the distribution of the frequency of appearance of the different symbol patterns, like the words in a text, follows a power-law distribution. If the n-tuples of symbols are sorted in the decreasing order, the frequency of appearance $\mathrm{N}_{\sigma(\mathrm{i})}$ of the n-tuple of rank $i$ in the sequence is given by the formula (1):

$$
N_{\sigma(i)}=k \cdot i^{\alpha}
$$

In this formula, $k$ and $\alpha$ are constants and the value of $\alpha$ characterizes the power law. In the distribution of words in English texts studied by Zipf, the value of $\alpha$ is close to 1. This law can be graphically represented in a bi-logarithmic scale diagram called Zipf plot. In this graphical plot, the least-square regression slope of the distribution enables an estimation of the power law exponent. Power-law distributions have been found in all natural languages as well as in different domains such as the distribution of city populations, Internet traffic or the repartition of DNA sequences in the human genome. The interest of Zipf law model is mainly based on pattern coding.

\subsection{Inverse Zipf Law}

G.K Zipf [14] also defined inverse Zipf law. It also deals with the statistical repartition of patterns frequency but, unlike the previously described Zipf law which emphasizes on the most frequent patterns in the sequence, the inverse Zipf law concerns the least frequent patterns in the sequence. According to inverse Zipf law, the number of words $I(f)$ which have the occurrence frequency $f$ is given by the formula (2):

$$
I(f)=a f^{-b}
$$

In this formula, $\mathrm{a}$ and $\mathrm{b}$ are constants and the value of $\mathrm{b}$ estimated by Zipf in his works on English texts is close to 2. This formulation has notably been used by Cohen et al. [6] for linguistic analysis. All these analysis tools designed primarily for text analysis can be adapted for use in different domains, in our case to image analysis. 


\section{Application to Image Analysis}

\subsection{Image Pattern Coding}

We can notice an image is a discrete representation in a 2 dimensional space. In order to use these models designed for text analysis in the domain of image analysis, we must first define an equivalent of the notion of word in the case of an image. We will work on image patterns, each pattern is defined as a $3 \times 3$ mask of adjacent pixels. Then we must define a pattern coding. If the grayscale levels were directly used, the frequency of appearance of each particular pattern would be very low due to the great number of possible patterns, and the distribution of pattern frequencies would not be statistically significant. So we must define some coding in order to reduce the number of possible patterns. A possibility is to divide the gray levels scale into a relatively small number of classes and to affect to each pixel the value of this class according to its luminance value. An example of pattern coding with this method is given on Fig. 1. The class $\mathrm{c}(\mathrm{x}, \mathrm{y})$ of a pixel with grayscale $\mathrm{g}(\mathrm{x}, \mathrm{y})$ would be given by the formula (3), where $N$ is the number of classes.

$$
c(x, y)=\operatorname{int}\left[\frac{N g(x, y)}{255}\right]
$$

This value allows a significant decrease of the maximal number of patterns from $256^{9}=4.7 \times 10^{21}$ to $9^{9}=3.87 \times 10^{8}$ and maintains consistency with human visual perception. With only 9 gray levels, the image structure is preserved and the main features are still clearly visible, as shown on Fig. 2.

\begin{tabular}{|c|c|c|}
\hline 255 & 210 & 210 \\
\hline 25 & 2 & 34 \\
\hline 40 & 2 & 40 \\
\hline
\end{tabular}

\begin{tabular}{|l|l|l|}
\hline 8 & 7 & 7 \\
\hline 0 & 0 & 1 \\
\hline 1 & 0 & 1 \\
\hline
\end{tabular}

Fig. 1. Original pattern (left) and pattern coded in 9 classes
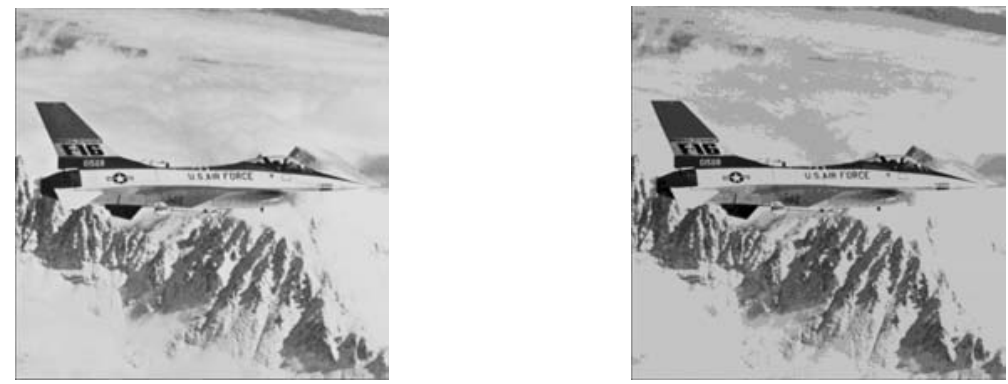

Fig. 2. Original image (left) and image coded with 9 gray levels (right) 


\subsection{Application of Power Law Models}

Both Zipf law and inverse Zipf law can be used to analyze an image. Analyzing an image with Zipf law consists in scanning the image with a 3x3 mask, coding the patterns, counting the frequency of appearance of each different pattern and sorting the patterns by decreasing order. The distribution of pattern frequencies with respect to their ranks can then be plotted in a bi-logarithmic scale diagram to visualize the actual law. In Fig. 3 is presented an image and the associated Zipf plot. For an image coded by partitioning the grayscale in 9 classes, it can be noticed that, using this coding the actual behavior of data would be better modelized using two different power laws. In fact two inner structures are put to the fore. One concerns the layout of regions and the other concerns the contours. The left part of the curve generally contains the most frequent patterns in which all the pixels belong to the same grayscale class, those patterns represents the homogenous zones of the image. The right part is made of patterns which pixels belong to different classes and represent contours and nonhomogenous zones, in photographic images they are considerably less frequent. As a consequence, the Zipf plot can be modelized by the least-square regression straight lines of the two parts of the curve. The inverse Zipf plot can also be used for analyzing an image. The patterns are coded in the same way as for the Zipf plot, the number of different patterns having each different frequency of appearance is counted, and the number of patterns with respect to their frequency is plotted in a bi-logarithmic scale diagram as shown in Fig. 4. We can notice that the left part, which represents the least frequent patterns, is linear. This result is in accordance with the results obtained by G.K. Zipf on texts. The distribution of pattern frequencies in an image follows a power law.
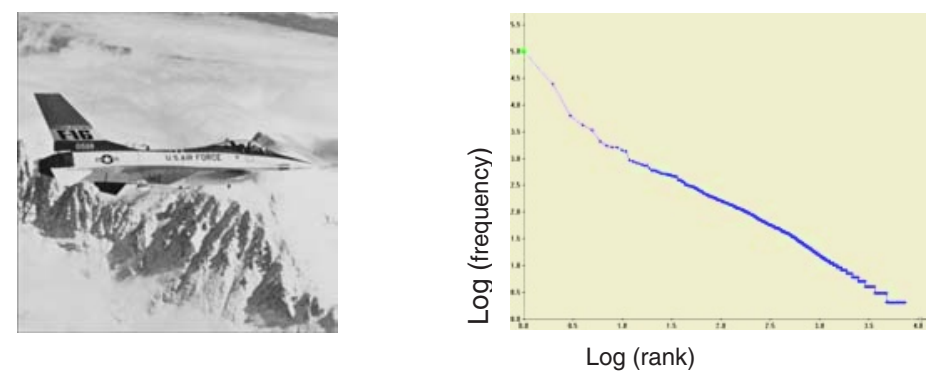

Fig. 3. Zipf plot (right) associated to the F-16 image (left) coded by partitioning the grayscale in 9 classes
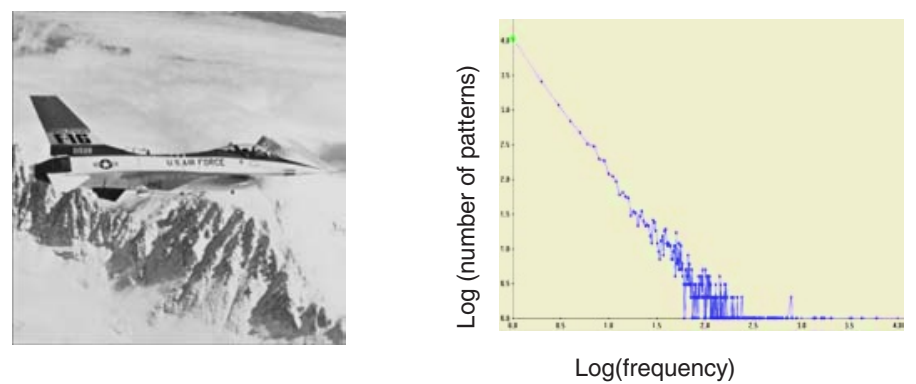

Fig. 4. Inverse Zipf plot for the F-16 image 


\section{Region of Interest Detection}

\subsection{Principle of the Method}

The objective is to detect regions of interest in images using power laws. The notion of region of interest is largely subjective, however some general considerations can be made for characterizing it. A region of interest can be defined as a region which has distinctive features from the rest of the image. It may be the foreground of the image, a region which appears less homogenous than the background, or a particular object in the image. In any case, it is a region which is different from the rest of the image. The use of power laws models will allow to find a region of the image which has a different distribution of texture pattern frequencies. The detection method consists in dividing the image into sub-images, computing the Zipf or the inverse Zipf distribution of each sub-image and classifying the sub-images according to the characteristics of this distribution. The size of the sub-images must be chosen properly, they must be large enough to have a statistically significant pattern distribution but also be small enough to allow a precise determination of the region of interest. The optimal size of the sub-images was determined experimentally, the best results were obtained for subimages containing about 5000 pixels and having the same aspect ratio as the initial image. Thus, the number of sub-images will be dependent on the image size.

\subsection{Use of Zipf Law}

As seen in Section 3, the regression lines of the two parts of the plot can represent the Zipf plot associated with an image. The classification method is based on the Zipf exponent that is to say on the regression slopes of the two parts of the plot. The Zipf plot associated with each sub-image of the image will be represented by a dot in the plane. In this representation the horizontal coordinate represents the slope of the left part of the Zipf plot corresponding to the homogenous patterns and the vertical coordinate represents the right part of the plot corresponding to the non-homogenous patterns. The points of this graph can be classified in two clusters according to their position with respect to the line of equation $\mathrm{y}=\mathrm{x}$. In most images, the points representing foreground objects tend to be situated just below the $\mathrm{y}=\mathrm{x}$ line, and more precisely in the left part of the cluster, at the left of a vertical line in close proximity with the cluster centroid. The equation of this vertical line was determined empirically to be:

$$
\mathrm{x}=\mathrm{G}_{\mathrm{x}} / 1.2
$$

In this formula, $G_{x}$ is the horizontal coordinate of the cluster center of gravity. The sub-images belonging to the region of interest are situated between the two lines, as indicated in Fig. 4. However, not all the points situated between the two lines represent sub-images belonging to the main region of interest. In order to deal with this problem, only the largest connected component of the sub-images corresponding to these points is kept in an automatic detection of a region of interest.

\subsection{Use of Inverse Zipf Law}

A similar classification method can also be used to detect region of interest with inverse Zipf law. The parameters of this classification will be the inverse Zipf exponent 
that is estimated by the slope of inverse Zipf plot, noted $b$ in Equation (2) and the number of unique patterns, noted $a$ in the same equation. The value of $a$ depends on the image size, so this parameter would only be significant if all the sub-images have the same size. The inverse Zipf plot associated with each sub-image will be represented by a dot in a graph with $\log (b)$ as the horizontal coordinate and $\log (a)$ as the vertical coordinate, as seen in Fig. 5.

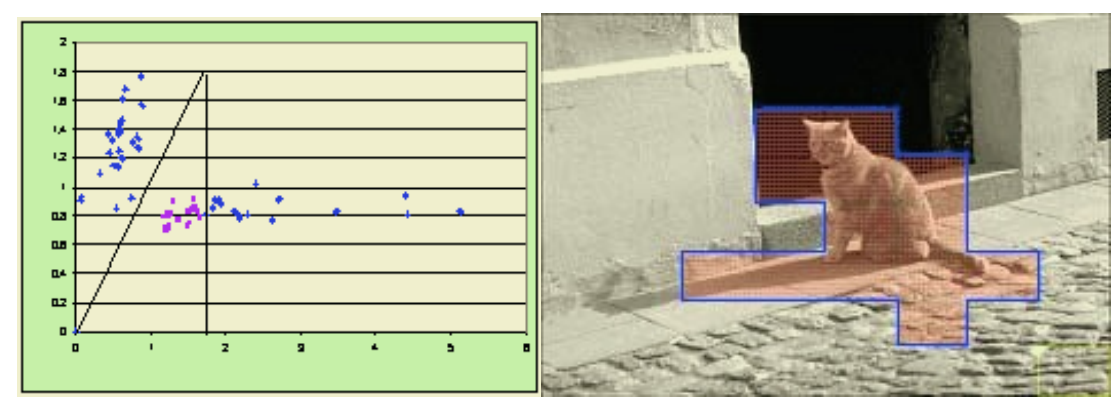

Fig. 4. Classification diagram for Zipf law (left) and corresponding region of interest (right)
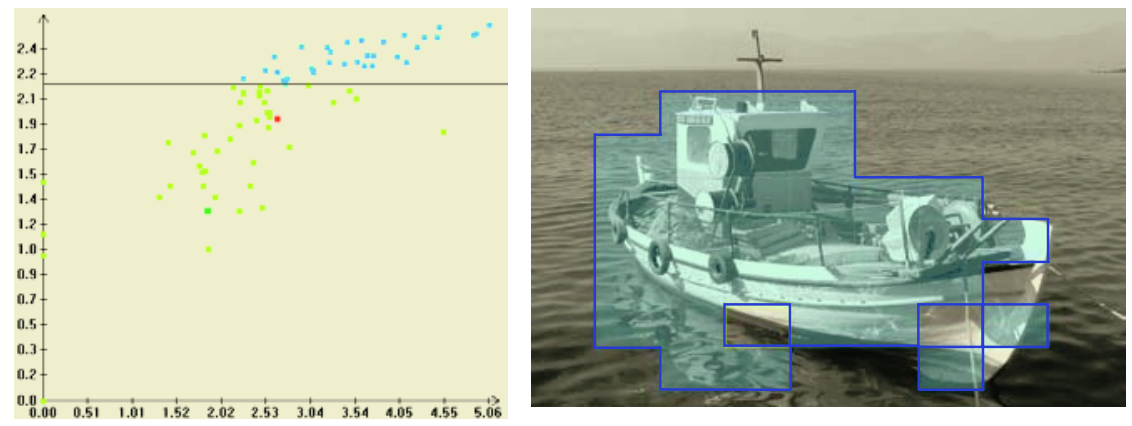

Fig. 5. Classification diagram for inverse Zipf law (left) and corresponding region of interest on the image(right)

In general, regions of interest tend to have more details than other parts of the image. Consequently, the sub-images associated with these regions will have more unique patterns than the rest of the image sub-images because they comprise more specific details and they will be situated at the top of the cluster. This part of the cluster may contain sub-images which do not belong to the region of interest. In that case, only the largest connected component is kept, as with Zipf law. The region of interest may contain holes; in that case we fill them by including the sub-images which have all the neighboring sub-images belonging to the region of interest. To ensure a better determination of the region of interest, the separation line between the two classes of the cluster can be determined dynamically, in such a way that the surface of the region of interest would always be between $20 \%$ and $50 \%$ of the total image surface. At first the direction of the separation line is chosen with respect to the inertia of the set of points and it intersects the center of gravity of the cluster. If the area of the region of interest is more than $50 \%$ of the surface area, after extracting the 
largest connected component and filling the holes, the separation line is raised iteratively by steps of 0.01 and the region of interest is recomputed until its surface area is $50 \%$ or less of the image surface area. Inversely, if the region of interest is less than $20 \%$ of the total image area, the separation line is lowered and the region of interest recomputed until it is at least $20 \%$ of the image surface area.

In some images, especially the images of artificial objects in natural backgrounds, the region of interest is more uniform than the background, and it is represented by points situated in the bottom part of the set of points. In that case the region of interest would be constituted by the sub-images represented by the points below the separation line. Thus we must make the distinction between the two types of images. The image is considered as having a region of interest more uniform than the background if the average proportion of unique patterns is higher than $50 \%$ in the whole image. This distinction has been confirmed by experimental results. This method allows to detect region of interest in various types of images, the results are generally consistent with human interpretation.

\section{Experimental results}

The two detection methods using Zipf and inverse Zipf law have been compared with each other to determine which of them detects the "best" region of interest, i.e. which one is the more consistent with human interpretation. An example of this comparison is shown on Fig. 6. In this image, the method using inverse Zipf law gives better results than the method using Zipf law, with Zipf law some parts of the object are classified as background and a significant part of the background is included in the region of interest. In most of the tested images, the inverse Zipf law gives better results than Zipf law for detecting objects of interest. The method has been tested in a set of 100 images of various subjects featuring a region of interest outlined by a human observer, the region of interest was correctly detected in $56 \%$ of the images with Zipf law and in $80 \%$ of the images with inverse Zipf law.
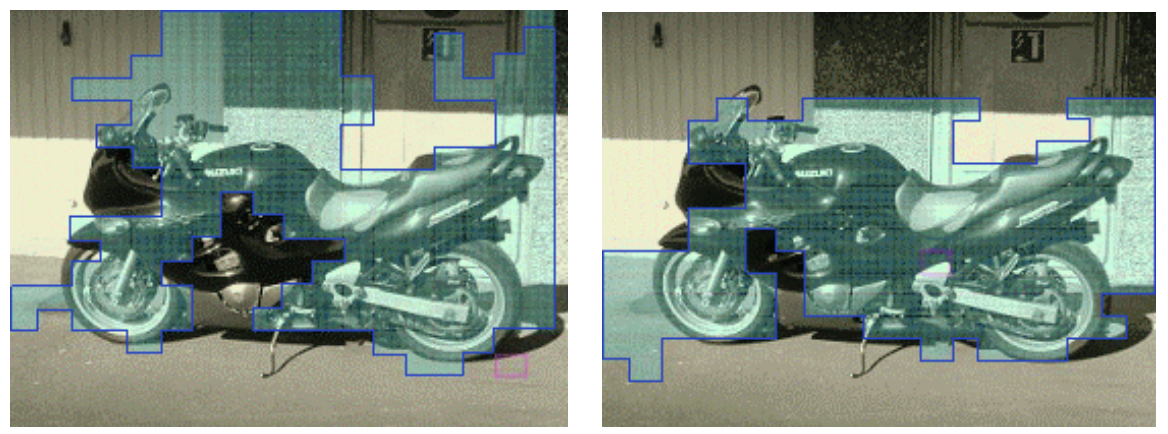

Fig. 6. Detection results with Zipf law (left) and inverse Zipf law (right)

It is also interesting to study the influence of the number of sub-images on the detection results. The image in Fig. 7 has been segmented in 8x8, 19x19 and 32x32 subimages, and the inverse Zipf detection method has been applied. When segmented in 
$8 \times 8$ sub-frames, the detection fails because the image is misclassified as having an object of interest more uniform than the background. In this case, it is the ground in front of the object which is detected. The segmentation in 19x19 sub-frames detects correctly the object of interest, and with the segmentation in 32x32 sub-frames, some uniform parts of the object are classified as parts of the background and textured background regions are classified as regions of interest. In most of the images, the best detection results are obtained when the surface of the sub-frames is around 5000 pixels, which correspond to $19 \times 19$ sub-images.
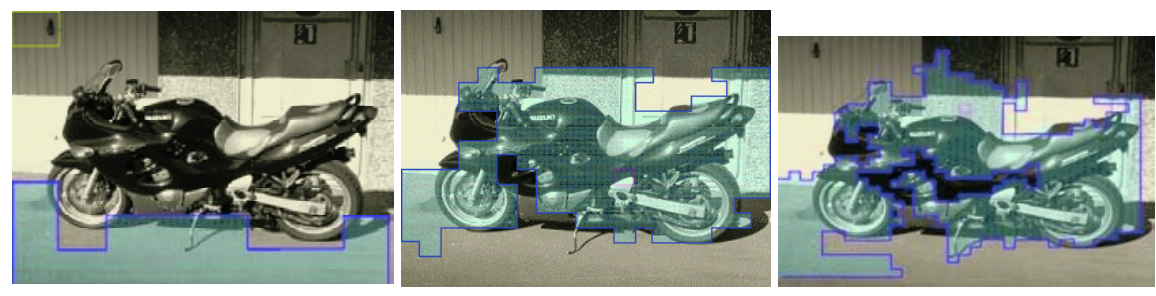

Fig. 7. Regions of interest detected with inverse Zipf law for a segmentation in $8 x 8$ (left), $19 \times 19$ (center) and 32x32 (right) sub-images.

\section{Conclusion}

The use of a method based on power laws allows the detection of regions of interest in an image without previous knowledge of the image or the nature of the region to be detected. Either Zipf law and inverse Zipf law can be used for region of interest detection. The classification of the sub-frames of the image in function of the characteristics of their representation by a power law can find a region of interest which is consistent with the human interpretation in terms of region of interest. Inverse Zipf law has better performances than Zipf law for region of interest detection. It finds more precise regions of interest, and it is able to detect regions of interest either when the region to be detected is more uniform than the image background or when it is less uniform. It is possible to find an optimal size for the sub-frames. The criteria used to classify the sub-frames are not the only possible, it is possible to use different criteria such as pattern frequency entropy. It will also be possible to use different pattern codings to improve the performances of the method.

\section{References}

1. Beaver, P., Quirk, S.M., Sattler, J.P.: Object Identification in Greyscale Imagery using Fractal Dimension, in M Novak (ed.): Fractal Reviews in the Natural and Applied Science, Chapman \& Hall, London, (1995) 63-73.

2. Kadir, T., Brady M.: Scale, Saliency and Image Description. International Journal of Computer Vision. Vol. 45 No. 2 (2001) 83-105

3. Wang, J.Z., Li J., Gray,R.M., Wiederhold,G.: Unsupervised Multiresolution Segmentation for Images with Low Depth of Field. IEEE Transactions on Pattern Analysis and Machine Intelligence. Vol. 23 No.1 (2001) 85-90 
4. Zipf, G.K.: Human Behavior and the Principle of Least Effort. Addison-Wesley, New York (1949)

5. Miller, G.A., Newman, E.B.: Test of a Statistical Explanation of the Rank-Frequency Relation for Words in Written English. American Journal of Psychology, 71 (1958) 209-218

6. Cohen, A. Mantegna, R.N., Havlin, S.: Numerical analysis of word frequencies in artificial and natural language texts. Fractals, Vol. 5 No.1 (1997) 95-104

7. Hill, B.M.: Zipf's law and prior distributions for the composition of a population. Journal of the American Statistical Association, 65 (1970) 1220-1232

8. Makse, H.A., Havlin, S., Stanley, H.E. Modelling urban growth patterns. Nature, 377 (1995) 608-612

9. Mantegna, R.N., Buldyrev, S.V., Goldberger, A.L., Havlin, S., Peng., C.K., Simons, M., Stanley, H.E.: Linguistic Features of Noncoding DNA Sequences, Phys. Rev. Lett. 73: 3169 (1994)

10. Breslau, L., Cao, P., Fan, L., Phillips, G., Shenker., S. Web caching and Zipf-like distributions: Evidence and implications. In Proceedings of IEEE Infocom 99 , New York (1999) $126-134$

11. Huberman, B.A., Pirolli, P., Pitkow, J., Lukose, R.: Strong Regularities in World Wide Web Surfing. Science, 280 (1998) 95-97

12. Vincent, N. Makris, P. Brodier, J.: Compressed Image Quality and Zipf's Law. In Proceedings of International Conference on Signal Processing (ICSP - IFICIAPRWCC2000), Beijing, China (2002) 1077-1084

13. Caron, Y., Makris, P., Vincent, N.: A method for detecting artificial objects in natural environmements. International Conference on Pattern recognition (ICPR - IAPR), Québec, Canada (2002), 600-603

14. Zipf, G.K. The Psychology of Language, an Introduction to Dynamic Philology, M.I.T. Press, Cambridge, Massachusetts (1965) 